\title{
Percepción de los estudiantes de la carrera de Gastronomía ante la experiencia de estudiar en modalidad virtual a consecuencia de las restricciones sanitarias por el COVID-19
}

\section{Perception of the students of the Gastronomy career before the experience of studying in virtual modality as a result of the sanitary restrictions due to COVID- 19}

\author{
GARCÍA-RAMÍREZ, Karina Nayeli† \& SANDOVAL-PALOMARES, Jessica*
}

Universidad Tecnológica de León, Blvd. Universidad Tecnológica \#225 Col. San Carlos CP. 37670 León, Gto. México.

ID $1^{\text {er }}$ Autor: Karina Nayeli, García-Ramírez / ORC ID: 0000-0002-7419-5846, Researcher ID Thomson: S-8644-2018, CVU CONACYT ID: 947600

ID $1^{\mathrm{er}}$ Coautor Jessica, Sandoval-Palomares / ORC ID: 0000-0002-3294-0916, Researcher ID Thomson: S-9841-2018, CVU CONACYT ID: 827848

DOI: $10.35429 / J P D .2020 .11 .4 .22 .28$

Recibido 25 de Enero, 2020; Aceptado 30 de Junio, 2020

\begin{abstract}
Resumen
Durante la contingencia causada por el COVID-19, las instituciones educativas en México tuvieron el reto de implementar de forma inesperada la educación a distancia en todos los niveles educativos, llevando a cabo distintas estrategias para aprender a utilizar distintas herramientas tecnológicas, con el propósito de rescatar el año escolar, el programa educativo y garantizar el aprendizaje de los estudiantes. Para la carrera de Gastronomía de la Universidad Tecnológica de León, el cambio a una modalidad virtual significó replantear el programa educativo, el desarrollo de las competencias a adquirir por el estudiantado, y adquirir habilidades técnico-profesionales propias de su formación. La presente investigación de corte cualitativa, proporciona una visión sobre la percepción del estudio en línea que tienen los alumnos durante el confinamiento, las estrategias utilizadas, el tipo de equipo y servicio de internet utilizado; los resultados proporcionan información valiosa y útil para implementar a corto plazo una serie de apoyos y propuestas de mejora que impacten en el proceso de aprendizaje y enseñanza.
\end{abstract}

\begin{abstract}
During the contingency caused by COVID-19, educational institutions in Mexico had the challenge of unexpectedly implementing distance education at all educational levels, carrying out different strategies to learn to use different technological tools, with the purpose to rescue the school year, the educational program and guarantee student learning. For the Gastronomy career at the Technological University of León, the change to a virtual modality meant rethinking the educational program, developing the skills to be acquired by students, and acquiring technicalprofessional skills typical of their training. This qualitative research provides an insight into the perception of the online study that students have during confinement, the strategies used, the type of equipment and internet service used; The results provide valuable and useful information to implement in the short term a series of supports and improvement proposals that impact the learning and teaching process.
\end{abstract}

COVID-19, Learning, Educational technology

COVID-19, Aprendizaje, Tecnología educativa.

Citación: GARCÍA-RAMÍREZ, Karina Nayeli \& SANDOVAL-PALOMARES, Jessica. Percepción de los estudiantes de la carrera de Gastronomía ante la experiencia de estudiar en modalidad virtual a consecuencia de las restricciones sanitarias por el COVID-19. Revista de Didáctica Práctica. 2020. 4-11:22-28.

\footnotetext{
*Correspondencia al Autor (Correo Electrónico: jspalomares@utleon.edu.mx)

$\dagger$ Investigador contribuyendo como primer autor.
} 


\section{Introducción}

La repentina migración a clases virtuales realizada por el distanciamiento social provocada por el COVID-19, significó para las instituciones educativas del país, establecer acciones y estrategias para atender a su comunidad, el profesorado se vio obligado a diseñar planeaciones de clase considerando la situación y en muchas ocasiones, las limitantes de sus estudiantes para acceder a internet; el alumnado por su parte, para adaptarse a la nueva circunstancia aprendió a utilizar la tecnología en pro de su formación, a realizar las actividades académicas prácticamente de forma autónoma, lo que les obliga a reflexionar y ser protagonistas de su propio aprendizaje.

Grynspan (2020), menciona que "al tiempo que se cerraban las puertas de las universidades, se abrían las puertas a las tecnologías", las tic's importantes antes, se convierten en las herramientas de excelencia para el abordaje de la educación, impactando en la continuidad y permanencia de las instituciones educativas de todos los niveles. Sin embargo, la brecha digital que existe en el país provoca que con dificultad las familias accedan con facilidad.

El impacto que la crisis del coronavirus COVID-19 ha provocado en las IES, generó que varias instancias nacionales e internacionales analizarán el fenómeno a profundidad, un ejemplo de lo anterior es el que se encuentra en sitio web de la Organización de las Naciones Unidas para la Educación, la Ciencia y la Cultura -UNESCO- en conjunto con la Secretaría General Iberoamericana SEGIB- y el Ministerio de Asuntos Exteriores de la Unión Europea; se menciona que el día 29 de junio del 2020, se realizó el "Diálogo sobre el impacto de la crisis del coronavirus COVID19 en las Instituciones de Educación Superior: respuestas a la emergencia y transformación digital de la Educación Superior".

La finalidad del encuentro fue la de reflexionar ante la necesidad de continuar con la actividad no presencial de las actividades académicas y del abordaje de una forma integral de la transformación digital, para potencializar y maximizar las tecnologías educativas, mitigando los efectos de la pandemia por el virus COVID-19 en la educación. Se analizaron cuatro ejes, a saber:
"Capacitación del profesorado y acceso de la comunidad universitaria a recursos para la enseñanza y el aprendizaje;

- Aseguramiento de la calidad de las enseñanzas virtuales;

- Equidad en el acceso y continuación de estudios superiores en la modalidad no presencial;

- Internacionalización de la Universidad en la actual situación de restricción de la movilidad internacional."

Las conclusiones, posterior a una profunda reflexión y análisis del impacto de la pandemia en la educación, se enfocan en los siguientes puntos:

"La necesidad de enfocar la cooperación iberoamericana en la capacitación del profesorado en las metodologías, pedagogías y apropiación de las tecnologías que permitan trasladar de la mejor forma posible lo que se hace en el aula presencial al aula virtual;

- La preocupación generalizada en torno al aseguramiento de la calidad en las enseñanzas virtuales;

- La necesidad de comenzar a abordar una movilidad virtual ante la imposibilidad de materializar movilidades con desplazamiento físico en el contexto de la actual pandemia".

La reflexión final de los especialistas gira en torno a la necesidad de implementar en las universidades proyectos de fortalecimiento de la educación a distancia, como son los planes de capacitación del profesorado, del personal administrativo y del estudiantado, incluyendo la necesaria inversión en infraestructura y tecnología. 
En la carrera de TSU en Gastronomía de la Universidad Tecnológica de León, la situación de la pandemia y el trabajo académico desde casa impactó principalmente en las clases prácticas, el estudiantado que antes de la contingencia llevaba a la práctica sus conocimientos teóricos en un laboratorio bajo el apoyo experto de un docente, ahora se veía en la necesidad de realizar la actividad desde la cocina de su casa, con los insumos y los materiales que se tienen en la alacena, realizando un gasto extra no considerado, desarrollando competencias y nuevas habilidades, entre las que se encuentran las siguientes: aprender a realizar video llamadas, video grabar con calidad, editar videos, explicar las recetas a una audiencia, entrevistar a personas, entre otras.

La presente investigación se realiza con la finalidad de analizar la percepción del estudiantado del tercer cuatrimestre de la carrera TSU en Gastronomía, del entorno actual y familiar, del desarrollo de sus habilidades y competencias, además del empleo de la tecnología durante su formación académica en la pandemia del COVID-19.

\section{Revisión de la literatura}

De acuerdo con la UNESCO, miles de estudiantes de todos los países del mundo dejaron de acudir a sus centros educativos debido al virus COVID-19; la pandemia mundial obligó a la comunidad académica internacional a explorar nuevas formas de enseñar y de aprender, incluidas las modalidades de educación a distancia y virtual.

Los cambios han resultado difíciles para los docentes y para los alumnos, quienes además de realizar las actividades académicas, deben enfrentarse a nuevos problemas de tipo emocional, físicos y un gasto económico no contemplado, situaciones que se presentaron a partir del confinamiento al que se vieron sujetos en el intento de frenar la propagación del virus en la población.
Al revisar la situación del estudiantado, se identifica presencia significativa de estrés ante el estudio, empleo de destrezas memorísticas versus aprendizaje significativo, la demanda para que se les indique con exactitud $y$ precisión qué hacer, cómo hacerlo y cuando hacerlo, baja utilización de su creatividad para realizar un proyecto o para entregar un trabajo, además de culpar al entorno, a la situación y a los demás cuando las cosas no les salen de la manera adecuada y esperada.

Hinojosa (2020), indica en su conferencia: la resiliencia académica en ambientes virtuales y a distancia, las acciones que deberá realizar el alumnado para tener éxito en su aprendizaje al continuar sus estudios en la modalidad línea:

a. Estar dispuesto a explorar y a experimentar, en especial, apertura para aprender de otras formas $y$ en otras modalidades.

b. Convertirse en estudiante proactivo, organizando el trabajo y su aprendizaje, controlando sus tiempos, aprender de forma autónoma el funcionamiento de las plataformas virtuales, con pensamiento crítico y lectura de comprensión para entender lo que le solicita el docente y qué se le debe enviar.

c. Realizar sus trabajos académicos de forma reflexiva, para dar sentido a lo que se lee y aprende, lo que le permitirá sentirse seguro con lo que se está estudiando.

d. Identificar las redes de apoyo establecidos por su institución y los apoyos que brindan los docentes; los cuales son fundamentales para aclarar dudas y recibir asesoría en general de la asignatura.

e. Reforzar actividades de aprendizaje colaborativo, con el fin de obtener una mejor calidad en el aprendizaje.

f. Actuar con ética y responsabilidad, evitando cometer plagio. 
Derivado de lo anterior, se generan las siguientes propuestas para fortalecer los ambientes académicos virtuales y a distancia:

1. La participación colaborativa fomenta la resiliencia comunitaria, esto significa que durante el trabajo en equipo se deberá identificar las fortalezas de cada estudiante en determinadas áreas o temas de una materia, para que compartan información con sus compañeros; de esta forma se pueden retroalimentar e ir solucionando juntos las dificultades.

2. Identificar las fortalezas y debilidades del estudiantado se convierte en una necesidad para la construcción de los grupos colaborativos.

3. El trabajo organizado y eficaz permite un buen resultado en las actividades académicas.

4. Manejo adecuado del estrés por el confinamiento, permitiendo una adecuada concentración en cuestiones académicas y organización de tiempo, evitado el retraso en la elaboración de los trabajos de las asignaturas.

\section{Método}

Investigación exploratoria, descriptiva, de tipo cualitativa; la información se obtuvo a través de un instrumento resuelto de forma virtual a través de la aplicación Forms de Microsoft, el cual está constituido por 6 interrogantes, para explorar la percepción de los estudiantes ante la situación de estudio en línea por la pandemia.

\section{Resultados}

La encuesta fue programada para ser resuelta por grupo de tercer cuatrimestre de la carrera de TSU en Gastronomía, se esperaban 30 respuestas, solo se obtuvieron 22, lo que corresponde al $73 \%$ de los estudiantes; a continuación, se mencionan los resultados obtenidos en cada una de las preguntas:

\section{Ámbito familiar:}

1. Durante el confinamiento, ¿Cómo se modificó tu dinámica familiar?
Las respuestas que brindaron los estudiantes se engloban en las siguientes afirmaciones:

- Mis padres creen que no tengo escuela y me piden hacer otras cosas en la casa.

- Respetan la hora de clase o asesoría, sin interrupciones o ruidos.

Primero me concentraba en mis estudios, después ayudaba a las tareas del hogar.

- Los roles no están claros, estamos en búsqueda de trabajo.

- $\quad$ Sin dificultad para adaptarnos.

Sin comunicación entre nosotros.

Frecuentes peleas entre nosotros.

$\mathrm{Al}$ analizar las respuestas, se observa que el espacio familiar requirió de adaptación para incluir el ámbito escolar, para alcanzar una cana convivencia y un respeto mutuo a las actividades que cada uno de los miembros de la familia realiza. Se destaca la valiosa oportunidad de incrementar la armonía, de conocerse más a fondo, y de establecer canales de comunicación; los alumnos se saben acompañados y respaldados tanto por sus familiares como por la universidad, lo que resulta en un resultado positivo de esta pandemia.

\section{Ámbito tecnológico:}

2. ¿Qué dispositivo utilizas para conectarte a clases virtuales?

Los resultados obtenidos muestran que el estudiantado que dio respuesta a la pregunta cuentan con un equipo que le permite la conexión a internet y por lo tanto, acceder a las clases virtuales. Se identifica que un $45 \%$ cuenta con equipo de cómputo portátil o de escritorio, el $44 \%$ un smartphone, el $11 \%$ cuenta con ambos dispositivos (celular y computadora); ninguno de los encuestados manifestó utilizar tabletas.

3. ¿Qué tipo de servicio de internet utilizas? 
Al analizar las respuestas en torno a la conectividad, los estudiantes informan lo siguiente: el $68 \%$ cuentan con servicio de internet en casa, el 14\% emplea los datos del smartphone, el $14 \%$ asiste a un ciber café para ingresar a clases, y un porcentaje pequeño, del $4 \%$ hace uso de la red contratada por un vecino.

Se destaca, posterior al análisis de las respuestas de la pregunta, el esfuerzo y el interés por el estudiantado por participar en las clases y cumplir con sus actividades académicas.

\section{Ámbito escolar:}

4. Durante el confinamiento:

\section{1 ¿Cómo describes tu experiencia escolar?}

Las respuestas que brindaron los estudiantes a la pregunta se analizaron y englobaron en las siguientes afirmaciones:

- $\quad$ No se aprende lo suficiente, la carrera no se presta para que sea realice en línea por las prácticas que se deben realizar.

- No les gusta a los estudiantes estar detrás de una pantalla, no es igual a estar presente.

- Es estresante, las dudas no se resuelven inmediatamente, y en ocasiones no se entiende bien lo que quiere el profesor.

La participación y la motivación es baja.

Es difícil, se cortan las videollamadas.

Se aprende a manejar la tecnología y las nuevas herramientas.

- $\quad$ No siempre se entiende a los profesores, y en ocasiones no dan respuesta a las dudas.
La dificultad que tienen los estudiantes ante la modalidad virtual implementada por la contingencia se percibe en las respuestas, los estudiantes manifiestan haber enfrentado un reto al adaptarse a estar detrás de una pantalla, previo a una formación académica totalmente presencial, la interacción con el profesor a través de un monitor es impersonal, impactando en el interés, motivación y atención a la sesión de clase; se identifica que en algunos casos no se cuenta con un espacio especial para tomar las clases, compartiéndolo con algún otro miembro de la familia; la dinámica de la familia se trastocó incrementando la dificultad para el aprendizaje.

4.2 ¿Qué has aprendido durante este tiempo?

Al indagar sobre el aprendizaje que se ha obtenido, es interesante los resultados obtenidos, y que a continuación se mencionan:

- Es importante adaptarse, los cambios cuestan trabajo y tiempo.

- No se ha aprendido nada académicamente.

Aprender a sobrevivir.

Ser paciente y tolerante.

Realizar las actividades sin importar el lugar.

Cumplir con lo que se solicita.

Actitud positiva, ser espontáneo.

Investigar, resolver las dudas por cuenta propia.

- Comunicación electrónica, empleo de tecnología.

Utilizar conocimientos adquiridos previamente. 
El confinamiento en casa por la contingencia ha dejado grandes aprendizajes en distintas áreas, impactando positivamente en las habilidades socioemocionales y el manejo de la frustración; las respuestas que se dieron a la pregunta nos deja claro que la juventud es resiliente, con una gran capacidad de adaptación, con interés en continuar con sus estudios a pesar de la adversidad; aprender a convivir y a compartir espacios no solo físicos, sino también virtuales.

5. ¿Qué sugerencias tienes para tus profesores, en pro de la mejora de las clases?

La pregunta tiene la finalidad de conocer que propuestas tienen los estudiantes para sus docentes, en la mejora de la actividad de clase, los resultados se mencionan a continuación:

- Que tengan claro su horario de clases.

- Estar más atentos a las dudas de los estudiantes.

Preparar clases con ejemplos, no solo teóricas.

Interactuar más con los estudiantes.

Tener mayor comunicación.

Ser más explícitos en las instrucciones de las actividades.

Atención pronta a los correos.

Tolerancia ante la dificultad de conectividad.

En el análisis de las respuestas, se destaca la necesidad de atención de los alumnos, y el sentido de lo impersonal que se percibe tomar clase a través del monitor. Resaltan las sugerencias en torno al respeto por los tiempos asignados y la necesidad de recibir instrucciones claras para elaborar las actividades.

\section{Conclusiones}

Sin duda, la pandemia por el Covid-19 provocó a nivel mundial, una época compleja para el entorno educativo; de un día al otro los estudiantes universitarios han dejado las aulas en las universidades para tomar sus clases en la sala o en el comedor de su casa; cambiaron a sus compañeros de carrera para compartir el espacio con sus familiares, modificando la dinámica familiar y los roles de sus integrantes.

Olivera (2020), comenta que "de la noche a la mañana los jóvenes universitarios han tenido que lidiar con una nueva forma de aprendizaje y realización de actividades $y$, sobre todo, con una forma inédita de convivencia académica y social que incide en su capacidad para aprender, debido al cambio sustancial en la modificación del proceso de enseñanza-aprendizaje".

Las respuestas que dieron los estudiantes al instrumento, proporcionan una visión de la adaptación y ajustes que han realizado, además de la necesidad de interactuar físicamente con sus pares y sus profesores dentro de las instalaciones de la universidad, y el significado que adquiere la institución educativa para los jóvenes, ya que para ellos se convierte en un segundo hogar, en un espacio de aprendizaje y de desarrollo integral.

Es evidente que el entorno familiar se ha modificado, se realiza un esfuerzo por mejorar la comunicación entre los integrantes y la convivencia diaria, genera una gran presión y un nivel de estrés que el joven por sí solo no sabe resolver, requiriendo por lo tanto de una red de apoyo que le brinde las herramientas necesarias, atendiendo su salud emocional y con impacto positivo en los procesos cognitivos.

En lo referente a las tecnologías y recursos digitales, se requiere que iniciativas como la que impulsa el gobierno del estado de Guanajuato, a través de Educafin, con la convocatoria de inclusión digital para estudiantes y docentes, asegura que la comunidad educativa de la región cuente con equipos de cómputo para acceder a las clases en la modalidad virtual. Un tema para resolver posteriormente será el de asegurar el acceso igualitario a internet, con conectividad efectiva. 
Como fue mencionado por los estudiantes, la calidad de la señal de internet afecta directamente al modelo y es causa de ausencias, desánimos y falta de motivación.

Meritorio es el trabajo docente, la suma de sus esfuerzos permitió la continuidad de los estudios en casa, sin embargo, es evidente la necesidad de capacitación del profesorado, reforzando su conocimiento en pedagogía digital, integración de las tecnologías de información al proceso de enseñanzaaprendizaje, además de brindar herramientas y estrategias para una comunicación efectiva y retroalimentación objetiva.

\section{Referencias}

CINVESTAV. (2020). El impacto de las herramientas digitales durante la pandemia. https://conexion.cinvestav.mx/Publicaciones/elimpacto-de-las-herramientas-digitales-durantela-pandemia

INEGI. (s.f.). Encuesta Nacional sobre Disponibilidad y Uso de TIC en Hogares. De 2015-2018: ENDUTIH.

Olivera, F. (2020). Percepción de estudiantes universitarios sobre la enseñanza en línea durante la pandemia de covid-19: algunos apuntes. Notas de coyuntura del crim No. 25, México, crim-unam, 6 pp.

Organización de las Naciones Unidas. (2020). COVID-19 y educación superior: El camino a seguir después de la pandemia. https://www.un.org/es/impacto-

académico/covid-19-y-educación-superior-elcamino-seguir-después-de-la-pandemia-0

Organización de las Naciones Unidas para la Educación, la Ciencia y la Cultura. (2020). Impacto de la crisis del coronavirus COVID-19 en las IES: respuestas a la emergencia y transformación digital de la educación superior.

https://www.iesalc.unesco.org/2020/07/17/dialo go-sobre-el-impacto-de-la-crisis-del-

coronavirus-covid-19-en-las-instituciones-deeducacion-superior-respuestas-a-la-emergenciay-transformacion-digital-de-la-educacionsuperior/
Organización de las Naciones Unidas para la Educación, la Ciencia y la Cultura. (2020). Volver a encauzar la educación: el aprendizaje digital durante la pandemia de COVID-19. https://es.unesco.org/news/volver-encauzareducacion-aprendizaje-digital-durantepandemia-covid-19

Quinteiro, Goris, J.A., (2020). Impacto de la crisis del coronavirus covid-19 en las IES: respuestas a la emergencia y transformación digital de la educación superior. 26/11/2020, de IESALC Sitio web: https://www.iesalc.unesco.org/2020/07/17/dialo go-sobre-el-impacto-de-la-crisis-delcoronavirus-covid-19-en-las-instituciones-deeducacion-superior-respuestas-a-la-emergencia$\mathrm{y}$-transformacion-digital-de-la-educacionsuperior/

Ramírez, I., Jaliri, C., Méndez Roca, J., Orlandini. I. (2020). ¿Cómo perciben los universitarios la educación virtual en tiempos de COVID-19? ASEFIE:

$\mathrm{http} / / /$ formacionib.org/noticias/?Comoperciben-los-universitarios-la-educacionvirtual-en-tiempos-de-COVID-19

Universidad Panamericana. (2020). La educación básica durante el covid-19: análisis pedagógico, psicológico y tecnológico. https://www.up.edu.mx/es/noticias/37847/laeducacion-basica-durante-el-covid-19-analisispedagogico-psicologico-y-tecnologico 